\title{
Fecal Short-Chain Fatty Acid Variations by Breastfeeding Status in Infants at 4 Months: Differences in Relative versus Absolute Concentrations
}

\author{
Sarah L. Bridgman', Meghan B. Azad'2, Catherine J. Field ${ }^{3}$, Andrea M. Haqq', \\ Allan B. Becker' ${ }^{2}$, Piushkumar J. Mandhane', Padmaja Subbarao ${ }^{4}$, Stuart E. Turvey ${ }^{5}$, \\ Malcolm R. Sears ${ }^{6}$, James A. Scott ${ }^{7}$, David S. Wishart ${ }^{8}$, Anita L. Kozyrskyj ${ }^{1 *}$ and \\ The CHILD Study Investigators ${ }^{9}$ \\ ${ }^{1}$ Department of Pediatrics, University of Alberta, Edmonton, AB, Canada, ${ }^{2}$ Department of Pediatrics and Child Health, \\ Children's Hospital Research Institute of Manitoba, University of Manitoba, Winnipeg, MB, Canada, ${ }^{3}$ Department of \\ Agricultural, Food and Nutritional Science, University of Alberta, Edmonton, AB, Canada, ${ }^{4}$ Department of Pediatrics, \\ University of Toronto, Toronto, ON, Canada, ${ }^{5}$ Department of Pediatrics, University of British Columbia, Vancouver, BC, \\ Canada, ${ }^{6}$ St Joseph's Healthcare and Department of Medicine, Firestone Institute for Respiratory Health, McMaster \\ University, Hamilton, ON, Canada, ${ }^{7}$ Dalla Lana School of Public Health, University of Toronto, Toronto, ON, Canada, \\ ${ }^{8}$ Department of Biological Sciences, University of Alberta, Edmonton, AB, Canada, ${ }^{9}$ Canadian Healthy Infant \\ Longitudinal Development Study, Hamilton, ON, Canada
}

Maria Carmen Collado, Consejo Superior de Investigaciones Cientificas (CSIC), Spain

Reviewed by: Miguel Gueimonde, Consejo Superior de Investigaciones Cientificas (CSIC), Spain Christophe Lacroix,

ETH Zurich, Switzerland

*Correspondence: Anita L. Kozyrsky] kozyrsky@ualberta.ca

Specialty section:

This article was submitted to Food Microbiology, a section of the journal

Frontiers in Nutrition

Received: 22 December 2016 Accepted: 24 March 2017 Published: 10 April 2017

Citation:

Bridgman SL, Azad MB, Field CJ, Haqq AM, Becker AB, Mandhane PJ, Subbarao P, Turvey SE, Sears MR, Scott JA, Wishart DS, Kozyrskyj AL and The CHILD Study Investigators (2017) Fecal Short-Chain Fatty Acid Variations by Breastfeeding Status in

Infants at 4 Months: Differences in Relative versus Absolute Concentrations.

Front. Nutr. 4:11

doi: 10.3389/fnut.2017.00011
Our gut microbiota provide a number of important functions, one of which is the metabolism of dietary fiber and other macronutrients that are undigested by the host. The main products of this fermentation process are short-chain fatty acids (SCFAs) and other intermediate metabolites including lactate and succinate. Production of these metabolites is dependent on diet and gut microbiota composition. There is increasing evidence for the role of SCFAs in host physiology and metabolic processes as well as chronic inflammatory conditions such as allergic disease and obesity. We aimed to investigate differences in fecal SCFAs and intermediate metabolites in 163 infants at 3-5 months of age according to breastfeeding status. Compared to no exposure to human milk at time of fecal sample collection, exclusive breastfeeding was associated with lower absolute concentrations of total SCFAs, acetate, butyrate, propionate, valerate, isobutyrate, and isovalerate, yet higher concentrations of lactate. Further, the relative proportion of acetate was higher with exclusive breastfeeding. Compared to non-breastfed infants, those exclusively breastfed were four times more likely (aOR 4.50, 95\% Cl 1.58-12.82) to have a higher proportion of acetate relative to other SCFAs in their gut. This association was independent of birth mode, intrapartum antibiotics, infant sex, age, recruitment site, and maternal BMI or socioeconomic status. Our study confirms that breastfeeding strongly influences the composition of fecal microbial metabolites in infancy.

Keywords: short-chain fatty acids, lactate, succinate, breastfeeding, infants, gut microbiota

\section{INTRODUCTION}

Gut microbiota have coevolved over millennia in a largely symbiotic relationship with the host. For the majority of time, human milk has been the sole source of nutrition for infants, providing essential nutrients for infant growth, as well as bioactive components to stimulate the gut microbiome. Both breastfeeding and gut microbial composition have been associated with a number of 
health outcomes during infancy and later life (1). In addition to providing a wide range of pathogen exclusion and immune and biosynthesis functions (2), a major role of gut microbiota is the metabolism of dietary fiber and other complex macronutrients that escape digestion in the small intestine.

The main products of nutrient breakdown by microbes are short-chain fatty acids (SCFAs), the predominant ones being acetate, butyrate, and propionate and to a lesser degree branchedchain fatty acids (BCFAs), valerate, isobutyrate, and isovalerate (3). Less well studied are lactate and succinate, intermediate metabolites in the microbial production of SCFAs (4). In adults, the majority of the SCFAs are rapidly absorbed or used as an energy source by colonocytes (5). There is increasing evidence that gut microbial metabolites have wider systemic effects in the host through their action as signaling molecules and involvement in regulators of gene expression $(6,7)$. SCFAs have been linked to appetite suppression by activating free fatty acid receptors in the intestine and increasing circulating anorectic gut hormones (3). They have also been shown to play an important role in the activation and differentiation of immune cells and have been implicated in inflammatory and allergic disease $(3,8,9)$.

The quantity and relative proportion of metabolites produced in the colon of adults has been closely linked to diet, as well as microbiota composition, diversity, and activity (10). Infancy represents a rapid period of gut microbial development, which is shaped by early-life events such as birth mode, antibiotic administration, and importantly, infant feeding (11-13). Human milk provides optimal nutrition for infants in the first 6 months of life and contains significant amounts of carbohydrates that escape digestion in the small intestine, identified as human milk oligosaccharides (HMOs), which are the preferred substrates for certain gut microbiota in the production of SCFAs (14). Studies have shown that infants who are exclusively breastfed have lower microbial diversity, with a predominance in Proteobacteria and Actinobacteria (most notably bifidobacteria), whereas formulafed infants tend to have a more diverse microbiota with increased abundance of Clostridia and Bacteroides species (15-17).

Gut microbiota dysbiosis and subsequent changes to metabolite profiles may be particularly important in infancy, which presents a critical window of opportunity in programming of future metabolic and immune health. Understanding how diet in early life can shape gut microbiota-associated metabolites is therefore of interest. While studies have previously reported differences in fecal SCFAs by infant diet (18-24), many had a small sample size, and few studies have reported on differences in relative proportions of SCFAs or intermediate metabolites. In addition, limited studies to date have assessed the impact of other birth factors, implicated in early microbiota development, on these associations (21).

We aimed to investigate differences in fecal SCFAs and intermediate metabolites in infants at 3-5 months of age according to breastfeeding. Specifically, we investigated whether fecal total and individual SCFAs, lactate, and succinate (measured as both absolute concentrations and relative proportions) differed according to breastfeeding status at the time of metabolite measurement and duration of exclusive breastfeeding. A secondary aim was to investigate whether these associations were independent of birth mode and intrapartum antibiotics use, as well as other early-life factors.

\section{MATERIALS AND METHODS}

\section{Study Design and Covariates}

The study included an unselected subset of 163 infants from the Canadian Healthy Infant Longitudinal Development national population-based birth cohort (25) (www.canadianchildstudy.ca) whose mothers were enrolled between November 2009 and February 2012 and who had fecal samples available for analysis collected between 3 and 5 months of age.

Mothers reported infant feeding practices using standardized questionnaires administered at 3, 6, and 12 months postpartum. Questionnaires asked mothers to record if they had ever breastfed their child, if they were currently breastfeeding their child, and the age of the child when breastfeeding ceased, if applicable. Mothers were also asked questions regarding formula feeding initiation and cessation. From these questionnaires, variables on feeding habits between birth and fecal sample collection were calculated. These included whether infants had ever been breastfed, duration of exclusive breastfeeding (never, $<3$ months, and $\geq 3$ months), and breastfeeding status at the time of fecal sample collection (exclusively breastfed, partially breastfed, and not breastfed).

Mode of birth [classified as vaginal, elective, or emergency cesarean section (CS)] and maternal intrapartum antibiotic prophylaxis (IAP) were extracted from hospital records. This was used to create a four-category variable for birth mode/IAP exposure: vaginal birth (no IAP), vaginal birth IAP, elective cesarean, and emergency cesarean, which we have previously reported on in association with infant gut microbiota (11). Information on other covariates including maternal age, ethnicity and education, infant sex, gestational age, and birth weight were obtained from hospital records or through standardized questionnaires completed by mothers. Maternal weight status [body mass index, weight (kilograms)/height (square meter)] was calculated from height and weight data taken from hospital records or measured at the 1-year postpartum clinic visit. There was a small amount of missing data for some variables (see Table 1).

\section{Sample Collection, Preparation, and Nuclear Magnetic Resonance (NMR) Analysis}

Samples were analyzed using NMR spectroscopy, which allows for the simultaneous measurement of a wide range of metabolites in a sample and has been successfully applied to metabolite measurement in fecal samples (26). Fecal samples (fresh or refrigerated for a short period) were collected using a standard protocol at a home visit at a mean age of 3.7 months (SD 0.47). Samples were refrigerated during transport and stored at $-80^{\circ} \mathrm{C}$ until analysis.

Approximately $100 \mathrm{mg}$ of fecal sample was homogenized and quickly transferred to an Eppendorf tube. One milliliter of ice cold water was added to the fecal powder and vortexed vigorously for $5 \mathrm{~min}$ followed by sonication at $4^{\circ} \mathrm{C}$ for $20 \mathrm{~min}$. The samples were further subjected to vortex shaking at $250 \mathrm{rpm}$ for $20 \mathrm{~min}$. The fecal water extract thus obtained was centrifuged 


\section{TABLE 1 | Study characteristics.}

\section{Characteristic}

\begin{tabular}{|c|c|}
\hline \multicolumn{2}{|l|}{ Mean (SD) } \\
\hline Maternal age (years) $(n=162)$ & $32(4.7)$ \\
\hline Gestational age (weeks) $(n=155)$ & $38.9(1.5)$ \\
\hline Birth weight $(g)(n=158)$ & $3,462(503)$ \\
\hline Age at stool sample collection (months), $n$ (\%) & $3.65(0.47)$ \\
\hline \multicolumn{2}{|l|}{ City of birth, $n(\%)$} \\
\hline Edmonton & $52(32)$ \\
\hline Vancouver & $95(58)$ \\
\hline Winnipeg & $16(10)$ \\
\hline \multicolumn{2}{|l|}{ Infant sex, $n(\%)$} \\
\hline Male & $90(55)$ \\
\hline Female & $73(45)$ \\
\hline \multicolumn{2}{|l|}{ Maternal ethnicity, $n(\%)(n=160)$} \\
\hline Caucasian & $119(74)$ \\
\hline Asian & $24(15)$ \\
\hline Other & $17(11)$ \\
\hline \multicolumn{2}{|l|}{ Birth mode, $n(\%)(n=158)$} \\
\hline Vaginal-no IAP & $78(49)$ \\
\hline Vaginal-IAP & $31(20)$ \\
\hline Cesarean section-emergency & $29(18)$ \\
\hline Cesarean section-elective & $20(13)$ \\
\hline \multicolumn{2}{|c|}{ Breastfeeding status at fecal sample collection, $n(\%)(n=158)$} \\
\hline None & $44(28)$ \\
\hline Partial & $66(42)$ \\
\hline Exclusive & $48(30)$ \\
\hline \multicolumn{2}{|l|}{ Ever breastfed, $n(\%)$} \\
\hline No & $17(10)$ \\
\hline Yes & $146(90)$ \\
\hline \multicolumn{2}{|l|}{ Exclusive breastfeeding duration, $n(\%)$} \\
\hline Never & $51(31)$ \\
\hline$<3$ months & $54(33)$ \\
\hline$\geq 3$ months & $58(36)$ \\
\hline
\end{tabular}

$N=163$ unless otherwise specified.

IAP, intrapartum antibiotic prophylaxis.

at $15,000 \times g$ for $1 \mathrm{~h}$ at $4^{\circ} \mathrm{C}$, and the supernatant was carefully aspirated into a fresh Eppendorf tube. This supernatant was centrifuged again at $15,000 \times g$ for $1 \mathrm{~h}$ at $4^{\circ} \mathrm{C}$ to precipitate any particulate fecal matter (that might have been introduced during the first separation), and the clear extract was transferred into a clean Eppendorf tube. The resultant fecal water was stored at $-20^{\circ} \mathrm{C}$ until further analysis.

A $570 \mu \mathrm{L}$ aliquot of fecal water was placed in a $1.5-\mathrm{mL}$ Eppendorf tube followed by the addition of $70 \mu \mathrm{L}$ of $\mathrm{D}_{2} \mathrm{O}$ and $60 \mu \mathrm{L}$ of standard buffer solution [585 mM NaHPO $4(\mathrm{pH} 7.0)$, $11.667 \mathrm{mM}$ disodium-2,2-dimethyl-2-silapentane-5-sulfonate (DSS), and $0.47 \% \mathrm{NaN}_{3}$ in $\mathrm{H}_{2} \mathrm{O}$ ]. The samples $(700 \mu \mathrm{L})$ were then transferred to a regular NMR tube for subsequent NMR spectral analysis.

All ${ }^{1} \mathrm{H}$-NMR spectra were collected on a Varian $500 \mathrm{MHz}$ Inova spectrometer equipped with a 5 -mm HCN Z-gradient pulsed-field gradient cyrogenic probe. ${ }^{1} \mathrm{H}-\mathrm{NMR}$ spectra were acquired at $25^{\circ} \mathrm{C}$ using the first transient of the Varian tnnoesy pulse sequence, which was chosen for its high degree of selective water suppression and quantitative accuracy of resonances around the solvent. Water suppression pulses were calibrated to achieve a bandwidth of 80 G. Spectra were collected with 128 transient and 8 steady-state scans using a 4-s acquisition time (48,000 complex points) and a 1-s recycle delay.

Before spectral analysis, all free induction decays were zerofilled to 64,000 data points and line broadened $0.5 \mathrm{~Hz}$. The methyl singlet produced by a known quantity of DSS was used as an internal standard for chemical shift referencing (set to $0 \mathrm{ppm}$ ) and for quantification. All ${ }^{1} \mathrm{H}-\mathrm{NMR}$ spectra were processed and analyzed using the Chenomx NMR Suite Professional software package version 8.1 (Chenomx Inc., Edmonton, AB, Canada). The Chenomx NMR Suite software allows for qualitative and quantitative analysis of an NMR spectrum by manually fitting spectral signatures from an internal database to the spectrum. Typically $90 \%$ of visible peaks were assigned to a compound, and more than $90 \%$ of the spectral area could be routinely fit using the Chenomx spectral analysis software. Most of the visible peaks were annotated with a compound name.

\section{Statistical Analysis}

Fecal metabolites were analyzed as absolute concentrations (micromoles per gram of feces) and as relative proportions (\%) of total SCFAs/BCFAs (labelled as SCFAs for short, and taken as the sum of acetate, butyrate, propionate, valerate, isobutyrate, and isovalerate). Median concentrations of total SCFAs (acetate, butyrate, propionate, valerate, isobutyrate, and isovalerate) and individual SCFAs as well as lactate and succinate were compared according to breastfeeding duration and breastfeeding at time of fecal sample collection using Kruskal-Wallis test (non-parametric ANOVA, with Bonferroni posttest for multiple comparison). Median relative proportions of SCFAs were also compared using Kruskal-Wallis test (with Bonferroni posttest).

Given the skewed nature of the data, fecal metabolites were categorized into a binary variable (high and low) using the median as a cut point, and associations with breastfeeding at stool collection were tested using logistic regression. Models were adjusted for birth mode and IAP exposure, age of stool sample collection as well as maternal and infant factors found to be associated with breastfeeding in our study (infant sex, city of birth, maternal education and BMI). All analysis was conducted using IBM SPSS (version 24). Statistical significance was considered when $P \leq 0.05$.

\section{RESULTS}

Fecal samples were collected from 163 infants between the ages of 3-5 months (mean age $3.65 \pm 0.47$ months). Subject characteristics of our sample are shown in Table 1. At fecal sample collection, $30 \%$ were exclusively breastfed and $42 \%$ of infants were partially breastfed. Twenty-two (14.2\%) infants had started solid foods. The majority of infants (69\%) were born vaginally, while almost a third of infants $(31 \%)$ were born by CS. Twenty-eight percent of vaginally delivered infants received IAP, and all cesarean infants received IAP as is standard practice in Canada.

Breastfeeding exclusivity at fecal sample collection differed by city of birth (Table 2). Males were less likely to be exclusively breastfed than females (25 versus $37 \%$ ), as were infants born to overweight or obese mothers and mothers with a lower level of 
TABLE 2 | Associations between breastfeeding and subject characteristics.

\begin{tabular}{|c|c|c|c|c|}
\hline \multirow[t]{2}{*}{ Characteristic } & \multicolumn{4}{|c|}{ Breastfeeding status, $N(\%)$} \\
\hline & None & Partial & Exclusive & $P$ value \\
\hline \multicolumn{5}{|l|}{ City of birth } \\
\hline Edmonton & $19(37)$ & $25(49)$ & $7(14)$ & 0.002 \\
\hline Vancouver & $18(20)$ & $35(38)$ & $39(42)$ & \\
\hline Winnipeg & $7(47)$ & $6(40)$ & $2(13)$ & \\
\hline \multicolumn{5}{|l|}{ Infant sex } \\
\hline Male & $22(25)$ & $44(50)$ & $22(25)$ & 0.06 \\
\hline Female & $22(31)$ & $22(31)$ & $26(37)$ & \\
\hline \multicolumn{5}{|l|}{ Maternal ethnicity $(n=155)$} \\
\hline Caucasian & $31(27)$ & $50(44)$ & $33(29)$ & 0.96 \\
\hline Asian & $6(25)$ & $10(42)$ & $8(33)$ & \\
\hline Other & $5(29)$ & $6(35)$ & $6(35)$ & \\
\hline \multicolumn{5}{|l|}{ Maternal education $(n=155)$} \\
\hline Less than University & $29(46)$ & $23(37)$ & $11(18)$ & $<0.001$ \\
\hline University or higher & $14(15)$ & $42(46)$ & $36(39)$ & \\
\hline \multicolumn{5}{|l|}{ Maternal BMI $\left(\mathrm{kg} / \mathrm{m}^{2}\right)(n=155)$} \\
\hline Normal $(<25)$ & $20(22)$ & 35 (39) & $36(40)$ & 0.02 \\
\hline Overweight or obese $(\geq 25)$ & $23(36)$ & $29(45)$ & $12(19)$ & \\
\hline \multicolumn{5}{|l|}{ Birth weight $(n=153)$} \\
\hline$\leq 4 \mathrm{~kg}$ & $38(29)$ & $54(41)$ & $41(31)$ & 0.45 \\
\hline$>4 \mathrm{~kg}$ & $5(25)$ & $11(55)$ & $4(20)$ & \\
\hline \multicolumn{5}{|l|}{ Gestational age $(n=150)$} \\
\hline Early term (<38 weeks) & $4(20)$ & $9(45)$ & $7(35)$ & 0.78 \\
\hline Term (38-40) & $33(30)$ & $45(41)$ & $32(29)$ & \\
\hline Late term $(41+$ weeks $)$ & $6(30)$ & $10(50)$ & $4(20)$ & \\
\hline \multicolumn{5}{|l|}{ Birth mode $(n=153)$} \\
\hline Vaginal-no IAP & $24(32)$ & $26(34)$ & $26(34)$ & 0.34 \\
\hline Vaginal-IAP & $5(17)$ & $16(55)$ & $8(28)$ & \\
\hline Cesarean section-emergency & $8(29)$ & $11(39)$ & $9(32)$ & \\
\hline Cesarean section-elective & $6(30)$ & $11(55)$ & $3(15)$ & \\
\hline
\end{tabular}

Breastfeeding status at fecal sample collection. $N=158$ unless otherwise specified.

$P$ value Pearson chi-square test.

$I A P$, intrapartum antibiotic prophylaxis.

education. Breastfeeding also differed according to birth mode, with exclusive breastfeeding at the time of fecal collection being highest in vaginally delivered infants not exposed to IAP and lowest in elective CS infants (34 and 15\%, respectively), although this difference was not statistically significant (chi-square $P=0.16$ ).

\section{Absolute Concentrations of SCFAs and Intermediate Metabolites and Breastfeeding Status}

Concentrations (micromoles per gram of feces) of SCFAs, lactate, and succinate in all infants are presented in Table 3. Total SCFA concentration was $142.0 \mu \mathrm{mol} / \mathrm{g}$ with the most abundant SCFA being acetate followed by propionate and butyrate.

In univariate analysis, infants who had ever been breastfed had lower concentrations of total SCFAs, acetate, butyrate, propionate, valerate, isobutyrate, and isovalerate and higher concentrations of lactate and succinate than those who had never been breastfed (Table 3). Compared to those not being breastfed at time of fecal metabolite profiling, those who were exclusively breastfed had significantly lower total SCFAs concentrations and

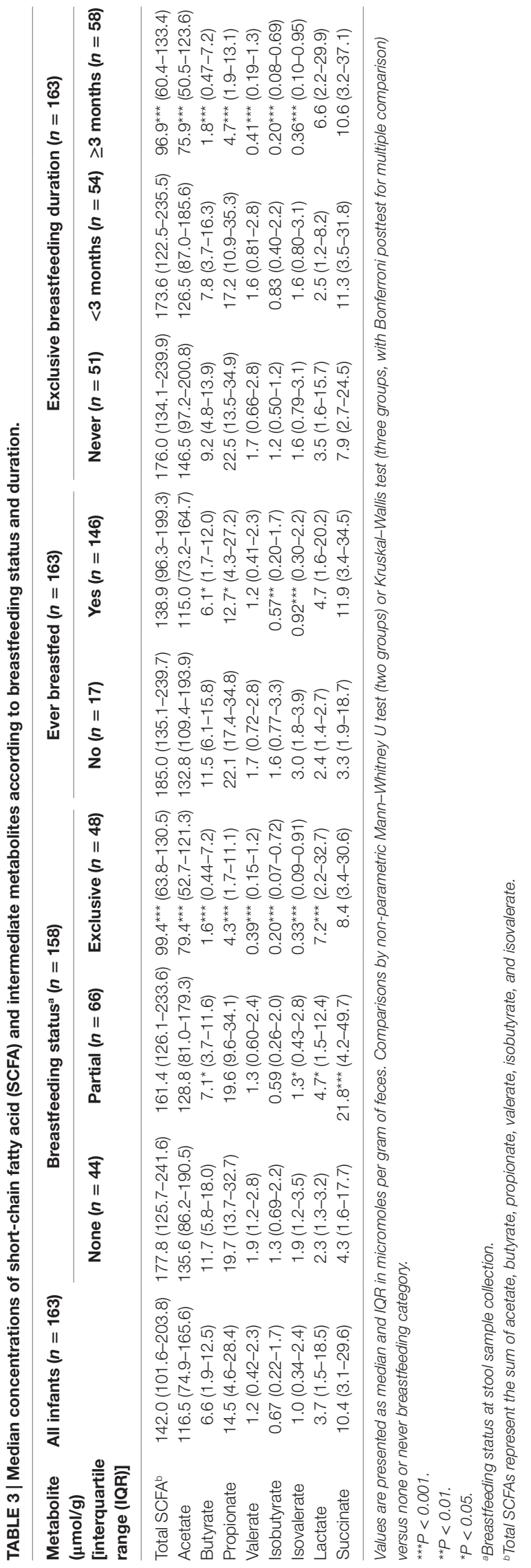


lower concentrations of all individual SCFAs (Table 3). Lactate was significantly higher in those exclusively breastfed versus those not breastfed $(7.2$ versus $2.3 \mu \mathrm{mol} / \mathrm{g} ; P<0.001)$. Those who were partially breastfed had similar concentrations of total SCFAs, acetate, and propionate compared to those not breastfed at the time of fecal metabolite profiling or concentrations falling midway between those not breastfed and those exclusively breastfed (seen for butyrate, valerate, isobutyrate, isovalerate, and lactate). Similar differences were observed according to duration of exclusive breastfeeding with those breastfed for $\geq 3$ months having significantly lower concentrations of SCFAs than infants who had never been breastfed (Table 3). Similar results were observed when data were restricted to infants who had not started solid foods (Table S1 in Supplementary Material) and vaginally delivered infants without exposure to IAP (Table S3 in Supplementary Material).

\section{Relative Proportions of SCFAs and Breastfeeding}

In all infants, acetate made up the largest proportion of the SCFAs $(80 \%)$ followed by propionate $(10 \%)$ and butyrate $(5 \%)$ with isovalerate being the least abundant (Table 4). In univariate analysis, infants exclusively breastfed at time of fecal metabolite profiling had significantly higher relative proportions of acetate and lower proportions of butyrate, propionate, isobutyrate, and isovalerate compared to those not breastfed (Table 4; Figure 1). Similar results were seen for those exclusively breastfed $\geq 3$ months versus those never exclusively breastfed (Table 4). Compared to infants who had never been breastfed, breastfed infants also tended to have higher proportions of acetate and lower proportions of butyrate, propionate, isobutyrate, and isovalerate although differences were only significant for the latter two metabolites. Similar trends were observed when data were restricted to infants who had not started solid foods (Table S2 in Supplementary Material) and infants vaginally delivered without exposure to IAP (Table S4 in Supplementary Material), although loss in statistical significance was observed in some instances.

The higher relative proportion of acetate seen in exclusively breastfed infants is in contrast to the lower overall concentration of acetate in fecal samples of these infants as illustrated in Figure 2.

\section{Odds of High SCFAs and Intermediate Metabolites According to Breastfeeding, Adjusted for Birth Mode, and IAP}

Exclusively breastfed infants had $86 \%$ lower odds of having high absolute concentrations of total SCFAs (concentrations greater than the group median) compared to those not breastfed at metabolite profiling [odds ratio (OR) 0.14 , 95\% CI 0.06-0.38; Table 5]. Odds of high total SCFAs was not significantly lower in those partially breastfed versus not breastfed (OR 0.92, 95\% CI 0.42-2.05), although partial breastfeeding was associated with reduced odds of having high butyrate, valerate, isobutyrate, and isovalerate. Exclusively breastfed infants also had significantly reduced odds of having high acetate, butyrate, propionate,

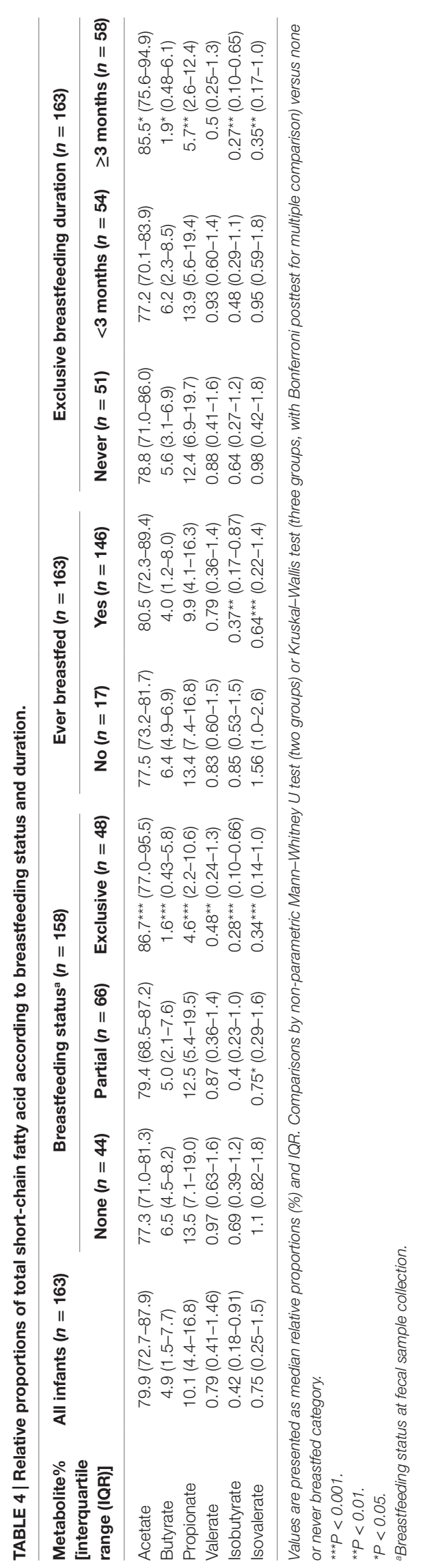




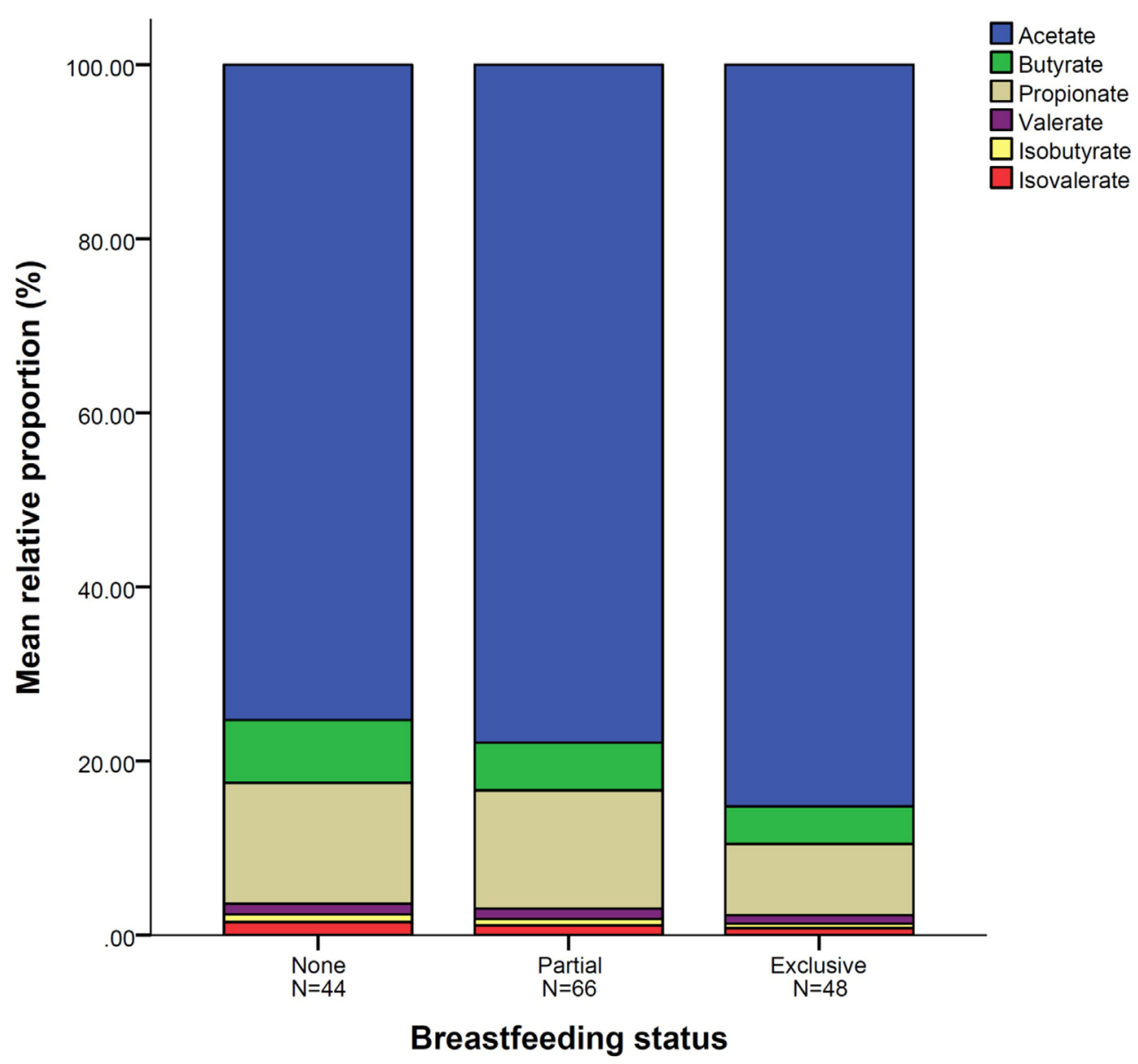

FIGURE 1 | Mean relative proportion of short-chain fatty acid according to breastfeeding status. $N=158$. Breastfeeding status at fecal sample collection.

valerate, isobutyrate, and isovalerate compared to those not breastfed (Table 5). Similar results were seen in these infants following adjustment for birth mode and IAP, as well as age at stool collection, sex, city of birth, maternal education, and BMI (Table 5).

Exclusively breastfed infants were seven times more likely to have high concentrations of lactate, and partially breastfed infants four times more likely than those not breastfed (OR 7.81, 95\% CI 2.99-20.37 and OR 4.56, 95\% CI 1.88-11.04, respectively; Table 5). These associations remained after adjustment for covariates. Partially breastfed infants, but not exclusively breastfed infants, had significantly higher odds of high succinate (OR 3.45, 95\% CI 1.53-7.79).

Infants exclusively breastfed were over four times more likely to have high relative proportions of acetate compared to those not breastfed (OR 4.26, 95\% CI 1.76-10.36; Table 6). This association was independent of birth mode and IAP, as well as other covariates (aOR 4.50, 95\% CI 1.58-12.82). In line with this, odds of high butyrate, propionate, valerate, isobutyrate, and isovalerate were significantly lower in exclusively breastfed infants. Associations with valerate did not remain after adjustment with all covariates. Partial breastfeeding was associated with reduced odds of high butyrate (OR $0.34,95 \%$ CI $0.15-0.80$ ), and isovalerate (OR 0.36 ,
95\% CI 0.16-0.81) although these associations were no longer significant after additional adjustment for maternal education and BMI.

\section{DISCUSSION}

In this subsample of 163 Canadian infants aged between 3 and 5 months from a general population birth cohort, we found strong associations between breastfeeding exclusivity and infant fecal metabolites. Exclusive breastfeeding was associated with lower absolute concentrations of total SCFAs, acetate, butyrate, propionate, valerate, isobutyrate, and isovalerate, yet higher concentrations of lactate. Further, the relative proportion of acetate was higher with exclusive breastfeeding. Compared to non-breastfed infants, those exclusively breastfed were four times more likely (aOR 4.50, 95\% CI 1.58-12.82) to have a higher proportion of acetate relative to other SCFAs in their gut. This association was independent of birth mode, intrapartum antibiotics, infant sex, age, recruitment site, and maternal BMI or socioeconomic status.

Our results are similar to that of Edwards et al. who also found a predominance in relative proportions of acetate (76\%) in breastfed infants at 4 weeks of age (19). Higher relative abundance 

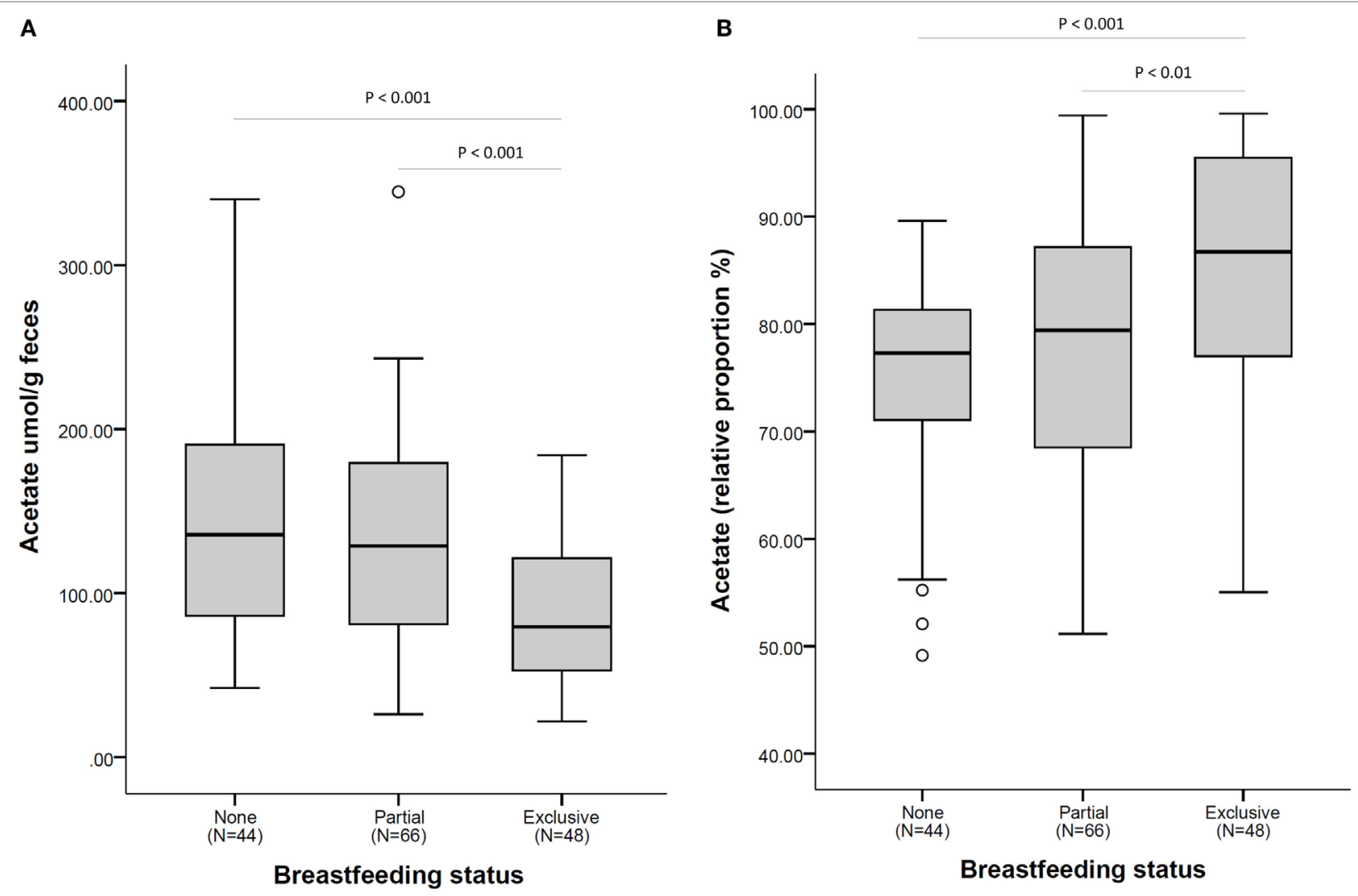

FIGURE 2 | Acetate fecal concentration (A) and as a relative proportion of total short-chain fatty acid (B), according to breastfeeding status at fecal sample collection. $N=158$. Comparisons by non-parametric Kruskal-Wallis test (with Bonferroni posttest for multiple comparison). Box plots present the group median (thick black line), upper quartile (top of box), and lower quartile (bottom of box). Whiskers present the maximum and minimum values excluding outliers (denoted by circles).

of acetate in exclusively breastfed infants may in part be due to the presence of HMOs in breast milk, not present in formula. HMOs, which represent the third largest component of breast milk, are soluble carbohydrates that are undigested by the host and provide substrates for select gut microbiota (27). Bifidobacterium species have been shown to be one of a narrow selection of gut bacteria that are able to metabolize HMO (28) and subsequently are overrepresented in the microbiota of breastfed infants compared to formula-fed infants $(11,16,29)$. Results from our group by Azad et al. found the Bifidobacteriaceae family to be enriched with breastfeeding (11). Bifidobacterium have been shown to metabolize HMO to produce acetate and lactate $(30,31)$. Martin et al. observed that increases in bifidobacteria counts corresponded to increases in fecal acetate (12). Although evidence is limited, higher acetate in breastfed infants may afford protection against intestinal pathogens and allergic disease (3). Fukudo et al. demonstrated that acetate, produced by bifidobacteria, improved intestinal defense and protected against Escherichia coli O157:H7 in mice (30). Thornburn et al. have proposed that acetate-mediated inhibition of histone deacetylases, demonstrated in an adult mouse model, increases transcription of the Foxp3 gene that may promote Treg-suppressing airway inflammation and inducing oral tolerance. In infants, Arrieta et al. found that reduced fecal acetate at 3 months was associated with allergic disease in later infancy (32).

We also observed lower absolute concentrations of SCFAs in exclusively breastfed infants, which are consistent with other published studies. In $111 \mathrm{fecal}$ samples analyzed by NMR, Martin et al. found lower concentrations of SCFAs in breastfed infants at 3 and 6 months born to overweight or obese mothers (20). In a small study on 4 infants using GC and LC mass spectrometry, valerate and isovalerate concentrations were higher in formula-fed infants, the latter over 40 times higher than breastfed infants (18). A study of 67 infants not only found lower fecal SCFA concentrations in breastfed infants but also observed that the addition of milk (formula or cows milk) to the diet of breastfed infants was sufficient to change the SCFA profile (22). We observed similar results, in that partially breastfed infants had SCFA and lactate concentrations more similar to exclusively formula-fed infants than infants exclusively breastfed.

The higher absolute concentrations of SCFAs observed in formula-fed infants may be a consequence of greater bacterial diversity observed in these infants in comparison to breastfed fed infants (11) and thus increased capability to metabolize substrates present in the gut. Indeed, previous results from our group found breastfeeding exclusivity to be inversely associated 
TABLE 5 | Associations between breastfeeding status and high metabolite concentrations, adjusting for birth mode and IAP, and other covariates.

\begin{tabular}{|c|c|c|c|c|c|c|}
\hline \multirow{2}{*}{$\begin{array}{l}\text { Breastfeeding } \\
\text { status }\end{array}$} & \multirow{2}{*}{$\begin{array}{l}\text { Number of infants } \\
\text { with high metabolite } \\
\text { concentration, } n / N(\%) \\
\text { ( } n=158)\end{array}$} & \multirow{2}{*}{$\begin{array}{l}P \text { value for } \\
\text { trend }^{\star}\end{array}$} & \multicolumn{4}{|c|}{ Odds of high metabolite concentration } \\
\hline & & & $\begin{array}{l}\text { Unadjusted OR (95\% CI) } \\
\qquad(n=153)\end{array}$ & $\begin{array}{l}\text { Model } 1 \text { aOR }(95 \% \mathrm{Cl}) \\
(n=153)\end{array}$ & $\begin{array}{l}\text { Model } 2 \text { aOR }(95 \% \mathrm{Cl}) \\
(n=153)\end{array}$ & $\begin{array}{c}\text { Model } 3 \text { aOR }(95 \% \mathrm{Cl}) \\
(n=148)\end{array}$ \\
\hline \multicolumn{7}{|l|}{ Total SCFA } \\
\hline None & 28/44 (63.6) & $<0.001$ & 1.00 (ref) & 1.00 (ref) & 1.00 (ref) & 1.00 (ref) \\
\hline Partial & 41/66 (62.1) & & $0.92(0.42-2.05)$ & $0.89(0.40-2.01)$ & $0.79(0.34-1.83)$ & $0.73(0.30-1.75)$ \\
\hline Exclusive & 9/48 (18.8) & & $0.14(0.06-0.38)$ & $0.14(0.05-0.36)$ & $0.13(0.05-0.38)$ & $0.13(0.04-0.39)$ \\
\hline \multicolumn{7}{|l|}{ Acetate } \\
\hline None & 25/44 (56.8) & 0.01 & 1.00 (ref) & 1.00 (ref) & 1.00 (ref) & 1.00 (ref) \\
\hline Partial & 43/66 (65.2) & & $1.38(0.62-3.05)$ & $1.45(0.64-3.30)$ & 1.27 (0.54-3.00) & $1.33(0.54-3.28)$ \\
\hline Exclusive & $14 / 48(29.2)$ & & $0.32(0.13-0.75)$ & $0.29(0.12-0.71)$ & $0.30(0.11-0.79)$ & $0.30(0.11-0.86)$ \\
\hline \multicolumn{7}{|l|}{ Butyrate } \\
\hline None & $33 / 44(75.0)$ & $<0.001$ & 1.00 (ref) & 1.00 (ref) & 1.00 (ref) & 1.00 (ref) \\
\hline Partial & $37 / 66(56.1)$ & & $0.37(0.15-0.87)$ & $0.37(0.16-0.90)$ & $0.35(0.14-0.87)$ & $0.42(0.16-1.11)$ \\
\hline Exclusive & $13 / 48(27.1)$ & & $0.11(0.04-0.28)$ & $0.11(0.04-0.29)$ & $0.12(0.04-0.35)$ & $0.13(0.04-0.39)$ \\
\hline \multicolumn{7}{|l|}{ Propionate } \\
\hline None & $33 / 44(75.0)$ & $<0.001$ & 1.00 (ref) & 1.00 (ref) & 1.00 (ref) & 1.00 (ref) \\
\hline Partial & $40 / 66(60.6)$ & & $0.50(0.22-1.17)$ & $0.45(0.18-1.09)$ & $0.42(0.17-1.05)$ & $0.48(0.18-1.25)$ \\
\hline Exclusive & $8 / 48(16.7)$ & & $0.07(0.03-0.20)$ & $0.68(0.02-0.18)$ & $0.06(0.02-0.19)$ & $0.07(0.02-0.24)$ \\
\hline \multicolumn{7}{|l|}{ Valerate } \\
\hline None & $32 / 44(72.7)$ & $<0.001$ & 1.00 (ref) & 1.00 (ref) & 1.00 (ref) & 1.00 (ref) \\
\hline Partial & $33 / 66(50.0)$ & & $0.32(0.14-0.75)$ & $0.31(0.13-0.74)$ & $0.35(0.14-0.86)$ & $0.42(0.16-1.07)$ \\
\hline Exclusive & $12 / 48(25.0)$ & & $0.11(0.04-0.28)$ & $0.10(0.04-0.27)$ & $0.13(0.04-0.36)$ & $0.15(0.05-0.45)$ \\
\hline \multicolumn{7}{|l|}{ Isobutyrate } \\
\hline None & $34 / 44$ (77.3) & $<0.001$ & 1.00 (ref) & 1.00 (ref) & 1.00 (ref) & 1.00 (ref) \\
\hline Partial & $31 / 66(47.0)$ & & $0.25(0.11-0.59)$ & $0.23(0.09-0.55)$ & $0.18(0.07-0.47)$ & $0.23(0.09-0.63)$ \\
\hline Exclusive & 15/48 (31.3) & & $0.13(0.05-0.34)$ & $0.13(0.05-0.35)$ & $0.12(0.04-0.33)$ & $0.15(0.05-0.45)$ \\
\hline \multicolumn{7}{|l|}{ Isovalerate } \\
\hline None & $34 / 44(77.3)$ & $<0.001$ & 1.00 (ref) & 1.00 (ref) & 1.00 (ref) & 1.00 (ref) \\
\hline Partial & $34 / 66$ (51.5) & & $0.30(0.13-0.72)$ & $0.30(0.12-0.71)$ & $0.25(0.10-0.63)$ & $0.33(0.13-0.86)$ \\
\hline Exclusive & $11 / 25(22.9)$ & & $0.08(0.03-0.23)$ & $0.09(0.03-0.23)$ & $0.08(0.03-0.22)$ & $0.09(0.03-0.29)$ \\
\hline \multicolumn{7}{|l|}{ Lactate } \\
\hline None & $10 / 44(22.7)$ & $<0.001$ & 1.00 (ref) & 1.00 (ref) & 1.00 (ref) & 1.00 (ref) \\
\hline Partial & $36 / 66(54.5)$ & & $4.56(1.88-11.04)$ & $4.51(1.82-11.18)$ & $5.75(2.16-15.26)$ & $5.37(1.90-15.15)$ \\
\hline Exclusive & $32 / 48(66.7)$ & & 7.81 (2.99-20.37) & $8.81(3.27-23.72)$ & $13.94(4.39-44.24)$ & $12.29(3.64-41.46)$ \\
\hline \multicolumn{7}{|l|}{ Succinate } \\
\hline None & $15 / 44(34.1)$ & 0.15 & 1.00 (ref) & 1.00 (ref) & 1.00 (ref) & 1.00 (ref) \\
\hline Partial & $41 / 66$ (62.1) & & $3.45(1.53-7.79)$ & $3.25(1.42-7.41)$ & $3.03(1.30-7.02)$ & $3.21(1.31-7.88)$ \\
\hline Exclusive & 24/48 (50.0) & & $2.07(0.88-4.90)$ & $2.03(0.85-4.84)$ & $1.85(0.72-4.72)$ & $1.93(0.71-5.22)$ \\
\hline
\end{tabular}

Breastfeeding status at fecal sample collection. High metabolite concentration denotes values above the median. Model 1: adjusted for birth mode and IAP; model 2: adjusted for birth mode and IAP, age of stool collection, sex, and city of birth; model 3: adjusted for birth mode and IAP, age of stool collection, sex, city of birth, maternal BMI, and education. OR, odds ratio; SCFA, short-chain fatty acid; IAP, intrapartum antibiotic prophylaxis.

*Pearson chi-square $P$ value for linear trend (univariate).

with both microbiota richness and diversity (11). Our finding may also be a result of differences in the composition or absorption of breast milk versus formula milk and thus differences in availability of substrates. Further, higher concentrations of branch-chain fatty acids, valerate, isobutyrate and isovalerate, derived from the metabolism of amino acids indicate reduced protein absorption or excess protein intake [potentially due to higher protein content of formula versus human milk (33)] in formula-fed infants. The availability of these substrates likely drive the higher abundance of proteolytic bacteria such as Bacteroides and Clostridia seen in formula-fed infants as reported in our study by Azad et al. (11). Higher concentrations of proteolytic metabolites in formula-fed infants may also be due to reduced carbohydrate availability in the absence of
HMOs and hence to greater derivation of energy from protein metabolism. Chow et al. demonstrated that when fermentable carbohydrates were not present in fecal cultures from both breast and formula-fed infants, metabolites indicative of protein fermentation were mainly produced; their production was reduced after the addition of various fermentable carbohydrate substrates similar to HMOs. Increased fecal SCFAs seen in formula-fed infants may have metabolic consequences. Several studies have reported greater concentrations of fecal SCFAs in overweight adults and children compared to lean counterparts (34-38) and correlations with other metabolic risk factors (38). Although causality is yet to be established, the authors of these studies hypothesize that higher SCFAs may reflect an increased capacity of the gut microbiota to harvest energy from the diet. 
TABLE 6 | Associations between breastfeeding status and high relative proportions of SCFA, adjusting for birth mode and IAP, and other covariates.

\begin{tabular}{|c|c|c|c|c|c|c|}
\hline \multirow{2}{*}{$\begin{array}{l}\text { Breastfeeding } \\
\text { status }\end{array}$} & \multirow{2}{*}{$\begin{array}{l}\text { Number of infants with } \\
\text { high relative proportion } \\
\text { of metabolite, } n / N(\%) \\
\qquad(n=158)\end{array}$} & \multirow{2}{*}{$\begin{array}{l}P \text { value for } \\
\text { trend }^{\star}\end{array}$} & \multicolumn{4}{|c|}{ Odds of high relative proportion of metabolite } \\
\hline & & & $\begin{array}{l}\text { Unadjusted OR }(95 \% \mathrm{Cl}) \\
\qquad(n=153)\end{array}$ & $\begin{array}{l}\text { Model } 1 \text { aOR }(95 \% \mathrm{Cl}) \\
\text { ( } n=153)\end{array}$ & $\begin{array}{l}\text { Model } 2 \text { aOR }(95 \% \mathrm{Cl}) \\
\qquad(n=153)\end{array}$ & $\begin{array}{c}\text { Model } 3 \text { aOR }(95 \% \mathrm{Cl}) \\
(n=148)\end{array}$ \\
\hline \multicolumn{7}{|l|}{ Acetate } \\
\hline None & 15/44 (34.1) & $<0.001$ & 1.00 (ref) & 1.00 (ref) & 1.00 (ref) & 1.00 (ref) \\
\hline Partial & 35/66 (53.0) & & $2.25(1.02-5.00)$ & $2.25(0.99-5.09)$ & $2.18(0.94-5.09)$ & $2.12(0.86-5.23)$ \\
\hline Exclusive & $34 / 48(70.8)$ & & $4.26(1.76-10.36)$ & $4.38(1.78-10.83)$ & $5.28(1.96-14.26)$ & $4.50(1.58-12.82)$ \\
\hline \multicolumn{7}{|l|}{ Butyrate } \\
\hline None & $32 / 44(72.7)$ & $<0.001$ & 1.00 (ref) & 1.00 (ref) & 1.00 (ref) & 1.00 (ref) \\
\hline Partial & $34 / 66(51.5)$ & & $0.34(0.15-0.80)$ & $0.33(0.14-0.78)$ & $0.35(0.14-0.87)$ & $0.51(0.19-1.35)$ \\
\hline Exclusive & 16/48 (33.3) & & $0.17(0.07-0.42)$ & $0.17(0.07-0.43)$ & $0.17(0.06-0.47)$ & $0.22(0.07-0.65)$ \\
\hline \multicolumn{7}{|l|}{ Propionate } \\
\hline None & $27 / 44(61.4)$ & 0.001 & 1.00 (ref) & 1.00 (ref) & 1.00 (ref) & 1.00 (ref) \\
\hline Partial & $37 / 66(56.1)$ & & $0.79(0.36-1.73)$ & $0.79(0.35-1.79)$ & $0.68(0.29-1.59)$ & $0.72(0.29-1.77)$ \\
\hline Exclusive & $13 / 48(27.1)$ & & $0.26(0.11-0.63)$ & $0.24(0.10-0.60)$ & $0.21(0.08-0.56)$ & $0.25(0.09-0.71)$ \\
\hline \multicolumn{7}{|l|}{ Valerate } \\
\hline None & 25/44 (56.8) & 0.04 & 1.00 (ref) & 1.00 (ref) & 1.00 (ref) & 1.00 (ref) \\
\hline Partial & $32 / 66$ (48.5) & & $0.64(0.29-1.39)$ & $0.58(0.26-1.30)$ & $0.65(0.28-1.50)$ & $0.84(0.35-2.06)$ \\
\hline Exclusive & $17 / 48(35.4)$ & & $0.38(0.16-0.91)$ & $0.39(0.16-0.92)$ & $0.44(0.17-1.12)$ & $0.58(0.21-1.58)$ \\
\hline \multicolumn{7}{|l|}{ Isobutyrate } \\
\hline None & $30 / 44$ (68.2) & 0.002 & 1.00 (ref) & 1.00 (ref) & 1.00 (ref) & 1.00 (ref) \\
\hline Partial & 33/66 (50.0) & & $0.45(0.20-1.01)$ & $0.42(0.19-0.97)$ & $0.37(0.16-0.88)$ & $0.53(0.21-1.33)$ \\
\hline Exclusive & $17 / 48(35.4)$ & & $0.26(0.11-0.62)$ & $0.26(0.11-0.64)$ & $0.22(0.08-0.58)$ & $0.30(0.11-0.85)$ \\
\hline \multicolumn{7}{|l|}{ Isovalerate } \\
\hline None & $32 / 44$ (72.7) & $<0.001$ & 1.00 (ref) & 1.00 (ref) & 1.00 (ref) & 1.00 (ref) \\
\hline Partial & $31 / 66(47.0)$ & & $0.32(0.14-0.73)$ & $0.33(0.14-0.77)$ & $0.33(0.14-0.78)$ & $0.45(0.18-1.09)$ \\
\hline Exclusive & $13 / 48(27.1)$ & & $0.14(0.05-0.35)$ & $0.13(0.05-0.34)$ & $0.13(0.05-0.35)$ & $0.16(0.06-0.47)$ \\
\hline
\end{tabular}

Breastfeeding status at fecal sample collection. High metabolite proportion denotes values above the median. Model 1: adjusted for birth mode and IAP; model 2: adjusted for birth mode and IAP, age of stool collection, sex, and city of birth; model 3: adjusted for birth mode and IAP, age of stool collection, sex, city of birth, maternal BMI, and education. OR, odds ratio; SCFA, short-chain fatty acid; IAP, intrapartum antibiotic prophylaxis.

${ }^{*}$ Pearson chi-square $P$ value for linear trend (univariate).

In addition to SCFAs, we also measured concentrations of lactate and succinate. Both are important intermediates in the production of SCFAs (3). Succinate is primarily converted to propionate through the succinate pathway. Lactate, produced by many colonizers of the infant gut including bifidobacteria and lactobacilli, can also be converted to propionate through the acrylate pathway but is also an important substrate used in crossfeeding by lactate-utilizing bacteria in the production of SCFAs as demonstrated by Pham et al. (24). Similar to other studies $(18-20,22)$, we observed higher lactate concentrations in exclusively breastfed infants, which is indicative of the predominance of lactate-producing bacteria, Lactobacillus and bifidobacteria, in the gut of breastfed infants. Lactate production in the gut affects luminal $\mathrm{pH}$, and stool from breastfed infants typically has a lower fecal $\mathrm{pH}$ compared to formula-fed infants (5.8 versus 6.3-7.10) $(22,39)$. Studies in adults have suggested that accumulation of lactate in the gut may have undesirable health consequences including increased risk of ulcerative colitis; however, in infancy, it may be an important mechanism in preventing overgrowth of $\mathrm{pH}$-sensitive pathogenic bacteria, such as Enterobacteriaceae and Clostridia $(40,41)$. Lactate has also been shown to be important in modulating immune and inflammatory processes and maintaining gut barrier function through stimulation of enterocyte proliferation (42). We are the first to report on differences in fecal succinate in infants. Although understudied, succinate may be an important signaling molecule and has been shown to activate GPR91 on dendritic cells and thus may play a role in modulation of gut immunity and inflammation $(6,43)$. Unlike the other associations we observed, differences in succinate were only apparent in those partially breastfed. This observation may in part be explained by the higher abundance of Bacteroidetes observed in partially breastfed infants reported in our study infants by Azad et al (11). Bacteroidetes utilize the succinate pathway in the formation of propionate $(4,44)$.

One of the strengths of our study is the use of infants from a well-characterized general birth cohort, affording detailed information on birth and early-life characteristics. This, along with our relatively large sample size, enabled us to account for prenatal and postnatal factors shown to impact early gut microbiota development (most importantly birth mode and perinatal antibiotic exposure) in our estimates of associations between fecal metabolites and breastfeeding. While we adjusted for IAP, shown to be significantly associated with gut microbiota dysbiosis in early life (11), we did not additionally adjust for postnatal infant antibiotic use due to missing data in our sample. However, it has been shown that IAP is a good proxy for perinatal antibiotic use in infancy (11). One limitation of our study was the indirect measurement of luminal metabolites via analysis of fecal samples. Concentrations 


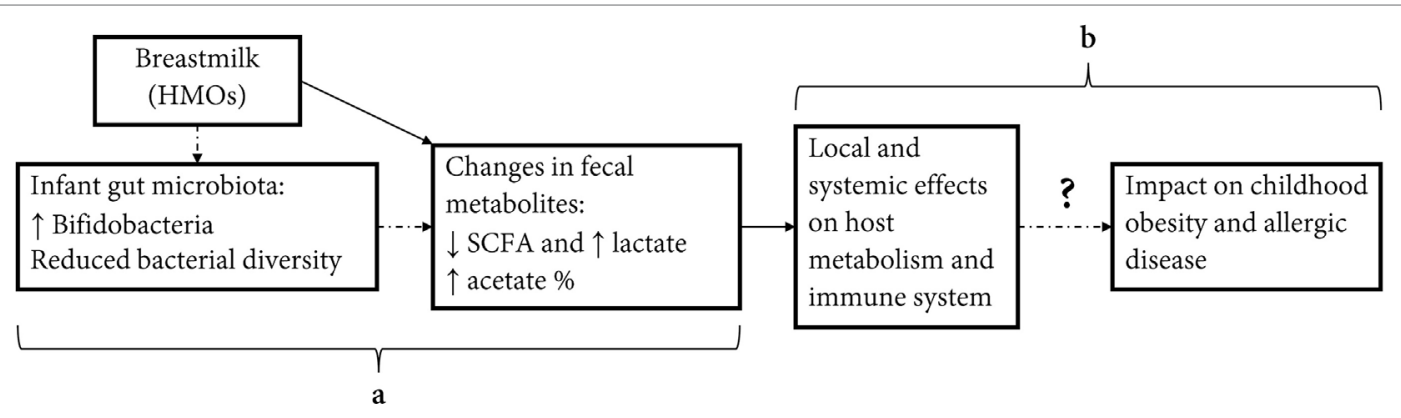

FIGURE 3 | Effect of infant diet on fecal short-chain fatty acids (SCFAs) and potential effect on host metabolic and immune programming. Human milk feeding causes changes in infant fecal metabolites, potentially through effects on gut microbiota composition (a). Changes in concentrations and relative proportions of SCFA and intermediate metabolites exert local effects on the gut environment and act as signaling molecules effecting host metabolism and immune system.

These actions may have important programming effects on inflammatory-mediated diseases in childhood, including obesity and allergic disease (b).

of metabolites in feces are a function not only of production but also of absorption, utilization by other microbes and stool transit time. Few studies have estimated whether fecal SCFA output is a reliable proxy for luminal content, particularly in early childhood although it is estimated that $95 \%$ of the SCFAs produced in the gut are rapidly absorbed with only $5 \%$ being excreted in the feces (45). In a study on healthy adults, Vogt and Wolever found fecal acetate to be inversely correlated to acetate absorption, suggesting that fecal acetate concentration may reflect intestinal absorption rather than production (46). However, given that the potential of analyzing fecal metabolites is to provide a biomarker to predict future disease risk, analysis of fecal samples provides a noninvasive and cost-effective method for use in epidemiological cohort studies. Our sampling was also only at one time point in infancy, and therefore, our data do not provide information on trends in metabolites over time in relation to diet.

In conclusion, exclusive breastfeeding has been associated with a number of beneficial health outcomes in early childhood including reduced infections, allergic disease, and improved metabolic markers (1). Recent research has highlighted the importance of our gut microbiota and associated metabolites as a potential causal factor or mediator in the programming of later disease states. Our study confirms that breastfeeding is strongly associated with gut metabolites, which may be an important mediator in the protective effect of breastfeeding on later-onset diseases (Figure 3). Given differences seen in the study between metabolites measured as absolute concentrations and relative proportions, we encourage future studies to report on both of these measures in relation to health outcomes.

\section{ETHICS STATEMENT}

Written informed consent was obtained from parents at enrollment. This study was approved by the University of Alberta, University of British Columbia and University of Manitoba, and McMaster University Human Research Ethics Boards.

\section{AUTHOR CONTRIBUTIONS}

AK, SB, MA, CF, AH, PM, and DW conceptualized the research question. SB analyzed the data and drafted the manuscript. SB and
AK interpreted findings and edited the manuscript. MA created breastfeeding measures for use in the analysis, contributed to interpretation of findings, and edited the manuscript. DW conducted NMR analysis on the fecal samples. JS, MS, AB, PM, PS, and ST directed the design and implementation of the CHILD study from which these samples were drawn. All authors revised and approved the final manuscript.

\section{ACKNOWLEDGMENTS}

The authors are grateful to all the families who took part in this study, and the whole CHILD team, which includes interviewers, nurses, computer and laboratory technicians, clerical workers, research scientists, volunteers, managers, and receptionists. The authors also thank Dr. Rupa Mandal and The Metabolomics Innovation Centre at the University of Alberta for conducting the fecal NMR profiling. The CHILD Study Investigators include the following: M. R. Sears (Director), McMaster University; P. Subbarao (Co-Director), The Hospital for Sick Children; S. S. Anand, McMaster University; M. Azad, University of Manitoba; A. B. Becker, University of Manitoba; A. D. Befus, University of Alberta; M. Brauer, University of British Columbia; J. R. Brook, University of Toronto; E. Chen, Northwestern University, Chicago; M. Cyr, McMaster University; D. Daley, University of British Columbia; S. Dell, Sick Children's Hospital; J. A. Denburg, McMaster University; Q. Duan, Queen's University; T. Eiwegger, The Hospital for Sick Children; H. Grasemann, Sick Children's Hospital; K. HayGlass, University of Manitoba; R. Hegele, Sick Children's Hospital; D. L. Holness, University of Toronto; P. Hystad, Oregon State University; M. S. Kobor, University of British Columbia; T. R. Kollman, University of British Columbia; A. L. Kozyrskyj, University of Alberta; C. Laprise, Université du Québec à Chicoutimi; W. Y. W. Lou, University of Toronto; J. Macri, McMaster University; P. J. Mandhane, University of Alberta; G. Miller, Northwestern University, Chicago; T. Moraes, Sick Children's Hospital; P. D. Paré, University of British Columbia; C. Ramsey, University of Manitoba; F. Ratjen, Sick Children's Hospital; A. Sandford, University of British Columbia; J. A. Scott, University of Toronto; J. Scott, University of Toronto; F. Silverman, University of Toronto; E. Simons, University of Manitoba; T. Takaro, Simon 
Fraser University; S. Tebbutt, University of British Columbia; T. To, Sick Children's Hospital; S. E. Turvey, University of British Columbia.

\section{FUNDING}

This research was specifically funded by the CIHR Canadian Microbiome Initiative (Grant No. 227312) and the Women and Children's Health Research Institute. The Canadian Institutes of

\section{REFERENCES}

1. Victora CG, Bahl R, Barros AJ, Franca GV, Horton S, Krasevec J, et al. Breastfeeding in the 21st century: epidemiology, mechanisms, and lifelong effect. Lancet (2016) 387:475-90. doi:10.1016/S0140-6736(15)01024-7

2. Sommer F, Backhed F. The gut microbiota - masters of host development and physiology. Nat Rev Microbiol (2013) 11:227-38. doi:10.1038/ nrmicro2974

3. Kumari M, Kozyrskyj AL. Gut microbial metabolism defines host metabolism: an emerging perspective in obesity and allergic inflammation. Obes Rev (2017) 18:18-31. doi:10.1111/obr.12484

4. Louis P, Flint HJ. Formation of propionate and butyrate by the human colonic microbiota. Environ Microbiol (2016) 1:1. doi:10.1111/1462-2920.13589

5. Bergman EN. Energy contributions of volatile fatty acids from the gastrointestinal tract in various species. Physiol Rev (1990) 70:567-90.

6. Koh A, De Vadder F, Kovatcheva-Datchary P, Backhed F. From dietary fiber to host physiology: short-chain fatty acids as key bacterial metabolites. Cell (2016) 165:1332-45. doi:10.1016/j.cell.2016.05.041

7. Morrison DJ, Preston T. Formation of short chain fatty acids by the gut microbiota and their impact on human metabolism. Gut Microbes (2016) 7:189-200. doi:10.1080/19490976.2015.1134082

8. Correa-Oliveira R, Fachi JL, Vieira A, Sato FT, Vinolo MA. Regulation of immune cell function by short-chain fatty acids. Clin Transl Immunol (2016) 5:e73. doi:10.1038/cti.2016.17

9. Richards JL, Yap YA, McLeod KH, Mackay CR, Marino E. Dietary metabolites and the gut microbiota: an alternative approach to control inflammatory and autoimmune diseases. Clin Transl Immunol (2016) 5:e82. doi:10.1038/ cti.2016.29

10. Flint HJ, Duncan SH, Scott KP, Louis P. Links between diet, gut microbiota composition and gut metabolism. Proc Nutr Soc (2015) 74:13-22. doi:10.1017/ S0029665114001463

11. Azad MB, Konya T, Persaud RR, Guttman DS, Chari RS, Field CJ, et al. Impact of maternal intrapartum antibiotics, method of birth and breastfeeding on gut microbiota during the first year of life: a prospective cohort study. BJOG (2016) 123:983-93. doi:10.1111/1471-0528.13601

12. Martin R, Makino H, Yavuz AC, Ben-Amor K, Roelofs M, Ishikawa E, et al. Early-life events, including mode of delivery and type of feeding, siblings and gender, shape the developing gut microbiota. PLoS One (2016) 11:e0158498. doi:10.1371/journal.pone.0158498

13. Yassour M, Vatanen T, Siljander H, Hamalainen AM, Harkonen T, Ryhanen SJ, et al. Natural history of the infant gut microbiome and impact of antibiotic treatment on bacterial strain diversity and stability. Sci Transl Med (2016) 8:343ra81. doi:10.1126/scitranslmed.aad0917

14. Bode L. The functional biology of human milk oligosaccharides. Early Hum $\operatorname{Dev}$ (2015) 91:619-22. doi:10.1016/j.earlhumdev.2015.09.001

15. Azad MB, Konya T, Maughan H, Guttman DS, Field CJ, Chari RS, et al. Gut microbiota of healthy Canadian infants: profiles by mode of delivery and infant diet at 4 months. CMAJ (2013) 185:385-94. doi:10.1503/cmaj.121189

16. Backhed F, Roswall J, Peng Y, Feng Q, Jia H, Kovatcheva-Datchary P, et al. Dynamics and stabilization of the human gut microbiome during the first year of life. Cell Host Microbe (2015) 17:690-703. doi:10.1016/j.chom.2015. 04.004

17. Penders J, Thijs C, Vink C, Stelma FF, Snijders B, Kummeling I, et al. Factors influencing the composition of the intestinal microbiota in early infancy. Pediatrics (2006) 118:511-21. doi:10.1542/peds.2005-2824
Health Research (CIHR) and the Allergy, Genes and Environment (AllerGen) Network of Centres of Excellence provided core support for the CHILD study.

\section{SUPPLEMENTARY MATERIAL}

The Supplementary Material for this article can be found online at http://journal.frontiersin.org/article/10.3389/fnut.2017.00011/ full\#supplementary-material.

18. Chow J, Panasevich MR, Alexander D, Vester Boler BM, Rossoni Serao MC, Faber TA, et al. Fecal metabolomics of healthy breast-fed versus formula-fed infants before and during in vitro batch culture fermentation. J Proteome Res (2014) 13:2534-42. doi:10.1021/pr500011w

19. Edwards CA, Parrett AM, Balmer SE, Wharton BA. Faecal short chain fatty acids in breast-fed and formula-fed babies. Acta Paediatr (1994) 83:459-62. doi:10.1111/j.1651-2227.1994.tb13059.x

20. Martin FP, Moco S, Montoliu I, Collino S, Da Silva L, Rezzi S, et al. Impact of breast-feeding and high- and low-protein formula on the metabolism and growth of infants from overweight and obese mothers. Pediatr Res (2014) 75:535-43. doi:10.1038/pr.2013.250

21. Midtvedt AC, Midtvedt T. Production of short chain fatty acids by the intestinal microflora during the first 2 years of human life. J Pediatr Gastroenterol Nutr (1992) 15:395-403. doi:10.1097/00005176-199211000-00005

22. Ogawa K, Ben RA, Pons S, de Paolo MI, Bustos Fernandez L. Volatile fatty acids, lactic acid, and $\mathrm{pH}$ in the stools of breast-fed and bottle-fed infants. J Pediatr Gastroenterol Nutr (1992) 15:248-52. doi:10.1097/00005176199210000-00004

23. Siigur U, Ormisson A, Tamm A. Faecal short-chain fatty acids in breast-fed and bottle-fed infants. Acta Paediatr (1993) 82:536-8. doi:10.1111/j.16512227.1993.tb12747.x

24. Pham VT, Lacroix C, Braegger CP, Chassard C. Early colonization of functional groups of microbes in the infant gut. Environ Microbiol (2016) 18:2246-58. doi:10.1111/1462-2920.13316

25. Subbarao P, Anand SS, Becker AB, Befus AD, Brauer M, Brook JR, et al. The Canadian Healthy Infant Longitudinal Development (CHILD) study: examining developmental origins of allergy and asthma. Thorax (2015) 70:998-1000. doi:10.1136/thoraxjnl-2015-207246

26. Matysik S, Le Roy CI, Liebisch G, Claus SP. Metabolomics of fecal samples: a practical consideration. Trends Food Sci Technol (2016) 57:244-55. doi:10.1016/j.tifs.2016.05.011

27. Garrido D, Dallas DC, Mills DA. Consumption of human milk glycoconjugates by infant-associated bifidobacteria: mechanisms and implications. Microbiology (2013) 159:649-64. doi:10.1099/mic.0.064113-0

28. Garrido D, Ruiz-Moyano S, Lemay DG, Sela DA, German JB, Mills DA. Comparative transcriptomics reveals key differences in the response to milk oligosaccharides of infant gut-associated bifidobacteria. Sci Rep (2015) 5:13517. doi:10.1038/srep13517

29. Harmsen HJ, Wildeboer-Veloo AC, Raangs GC, Wagendorp AA, Klijn N, Bindels JG, et al. Analysis of intestinal flora development in breast-fed and formula-fed infants by using molecular identification and detection methods. J Pediatr Gastroenterol Nutr (2000) 30:61-7. doi:10.1097/00005176200001000-00019

30. Fukuda S, Toh H, Hase K, Oshima K, Nakanishi Y, Yoshimura K, et al. Bifidobacteria can protect from enteropathogenic infection through production of acetate. Nature (2011) 469:543-7. doi:10.1038/nature09646

31. Matsuki T, Yahagi K, Mori H, Matsumoto H, Hara T, Tajima S, et al. A key genetic factor for fucosyllactose utilization affects infant gut microbiota development. Nat Commun (2016) 7:11939. doi:10.1038/ncomms11939

32. Arrieta MC, Stiemsma LT, Dimitriu PA, Thorson L, Russell S, Yurist-Doutsch S, et al. Early infancy microbial and metabolic alterations affect risk of childhood asthma. Sci Transl Med (2015) 7:307ra152. doi:10.1126/scitranslmed. aab2271

33. Mace K, Steenhout P, Klassen P, Donnet A. Protein quality and quantity in cow's milk-based formula for healthy term infants: past, present and 
future. Nestle Nutr Workshop Ser Pediatr Program (2006) 58:109-203. doi:10.1159/000095063

34. Payne AN, Chassard C, Zimmermann M, Muller P, Stinca S, Lacroix C. The metabolic activity of gut microbiota in obese children is increased compared with normal-weight children and exhibits more exhaustive substrate utilization. Nutr Diabetes (2011) 1:e12. doi:10.1038/nutd.2011.8

35. Fernandes J, Su W, Rahat-Rozenbloom S, Wolever TM, Comelli EM. Adiposity, gut microbiota and faecal short chain fatty acids are linked in adult humans. Nutr Diabetes (2014) 4:e121. doi:10.1038/nutd.2014.23

36. Rahat-Rozenbloom S, Fernandes J, Gloor GB, Wolever TM. Evidence for greater production of colonic short-chain fatty acids in overweight than lean humans. Int J Obes (Lond) (2014) 38:1525-31. doi:10.1038/ijo.2014.46

37. Schwiertz A, Taras D, Schafer K, Beijer S, Bos NA, Donus C, et al. Microbiota and SCFA in lean and overweight healthy subjects. Obesity (Silver Spring) (2010) 18:190-5. doi:10.1038/oby.2009.167

38. Teixeira TF, Grzeskowiak L, Franceschini SC, Bressan J, Ferreira CL, Peluzio MC. Higher level of faecal SCFA in women correlates with metabolic syndrome risk factors. Br J Nutr (2013) 109:914-9. doi:10.1017/S0007114512002723

39. Knol J, Scholtens P, Kafka C, Steenbakkers J, Gro S, Helm K, et al. Colon microflora in infants fed formula with galacto- and fructo-oligosaccharides: more like breast-fed infants. J Pediatr Gastroenterol Nutr (2005) 40:36-42. doi:10.1097/00005176-200501000-00007

40. Duncan SH, Louis P, Thomson JM, Flint HJ. The role of $\mathrm{pH}$ in determining the species composition of the human colonic microbiota. Environ Microbiol (2009) 11:2112-22. doi:10.1111/j.1462-2920.2009.01931.x

41. Sun Y, O'Riordan MX. Regulation of bacterial pathogenesis by intestinal short-chain fatty acids. Adv Appl Microbiol (2013) 85:93-118. doi:10.1016/ B978-0-12-407672-3.00003-4
42. Garrote GL, Abraham AG, Rumbo M. Is lactate an undervalued functional component of fermented food products? Front Microbiol (2015) 6:629. doi:10.3389/fmicb.2015.00629

43. Rubic T, Lametschwandtner G, Jost S, Hinteregger S, Kund J, Carballido-Perrig $\mathrm{N}$, et al. Triggering the succinate receptor GPR91 on dendritic cells enhances immunity. Nat Immunol (2008) 9:1261-9. doi:10.1038/ni.1657

44. Louis P, Hold GL, Flint HJ. The gut microbiota, bacterial metabolites and colorectal cancer. Nat Rev Microbiol (2014) 12:661-72. doi:10.1038/ nrmicro3344

45. den Besten G, van Eunen K, Groen AK, Venema K, Reijngoud DJ, Bakker BM. The role of short-chain fatty acids in the interplay between diet, gut microbiota, and host energy metabolism. J Lipid Res (2013) 54:2325-40. doi:10.1194/ jlr.R036012

46. Vogt JA, Wolever TM. Fecal acetate is inversely related to acetate absorption from the human rectum and distal colon. J Nutr (2003) 133:3145-8.

Conflict of Interest Statement: The authors declare that the research was conducted in the absence of any commercial or financial relationships that could be construed as a potential conflict of interest.

Copyright (C) 2017 Bridgman, Azad, Field, Haqq, Becker, Mandhane, Subbarao, Turvey, Sears, Scott, Wishart, Kozyrskyj and The CHILD Study Investigators. This is an open-access article distributed under the terms of the Creative Commons Attribution License (CC BY). The use, distribution or reproduction in other forums is permitted, provided the original author(s) or licensor are credited and that the original publication in this journal is cited, in accordance with accepted academic practice. No use, distribution or reproduction is permitted which does not comply with these terms. 